\title{
A novel visual test for hydrogen sulfide on the tongue dorsum*
}

\author{
Takashi Kanehira ${ }^{1}$, Hirohisa Hongo ${ }^{1}$, Junji Takehara ${ }^{1}$, Kozo Asano ${ }^{2}$, Kazumi Osada ${ }^{3}$, Hiroshi Izumi ${ }^{3}$, \\ Yoshihiro Fujii ${ }^{4}$, Wataru Sakamoto ${ }^{4,5 \#}$ \\ ${ }^{1}$ Division of Preventive Dentistry, Graduate School of Dental Medicine, Hokkaido University, Sapporo, Japan \\ ${ }^{2}$ Applied Microbiology, Applied Bioscience, Graduate School of Agriculture, Hokkaido University, Sapporo, Japan \\ ${ }^{3}$ Department of Physiology, School of Dentistry, Health Sciences University of Hokkaido, Ishikari, Japan \\ ${ }^{4}$ Institute of QOL, Fuji Women's University, Ishikari, Japan \\ ${ }^{5}$ Serotec Laboratory, Ebetsu, Japan \\ Email: ${ }^{*}$-sakamoto@serotec.co.jp
}

Received 29 August 2012; revised 29 September 2012; accepted 9 October 2012

\begin{abstract}
Objective: The purpose of this study was to develop a novel colorimetric system for direct detection of hydrogen sulfide on the tongue dorsum from subjects with complaints of halitosis. Method: An assay system comprised of a cotton swab impregnated with zinc sulfate, attached chromatography paper, stabilizing solution, and coloring reagents for the methylene blue technique was developed. Using this system we investigated on the visualization of the hydrogen sulfide on the solid phase and possibility for evaluating the origin of oral malodor on the tongue dorsum. Results: The assay system made possible visualization of a linear curve ranging from $5.3 \mathrm{ng}$ to $85 \mathrm{ng}$ of hydrogen sulfide, with low reagent consumption and miniaturization. However, the sensitivity was different for gaseous and fluid samples. The biofilm of the tongue dorsum from subjects with complaints of halitosis showed different shades of methylene blue depending on the concentration of hydrogen sulfide. A positive correlation was found between hydrogen sulfide on the tongue dorsum and the concentration of VSC hydrogen sulfide in mouth breath as measured using an Oral Chroma semiconductor gas sensor. Conclusion: The results of this study suggest that the novel assay system can be used as a visual warning sensor for halitosis by measuring hydrogen sulfide on the tongue dorsum.
\end{abstract}

Keywords: Halitosis; Tongue dorsum; VSC Hydrogen Sulfide; Methylene Blue

\footnotetext{
${ }^{*}$ This work was supported by JSPS KAKENHI funds from a Grant-inAid for Scientific Research (C) (No. 23593081) to T.K. There is no conflict of interest for any author.

${ }^{\#}$ Corresponding author.
}

\section{INTRODUCTION}

Oral malodor is a common problem that affects people in daily life. Most reports now agree that the most frequent sources of oral malodor exist within the oral cavity and include bacterial reservoirs such as the tongue dorsum, saliva and periodontal pockets, where anaerobic bacteria degrade sulphur-containing amino acids to produce foul smelling VSC (hydrogen sulfide, dimethyl sulfide, and methyl mercaptan) [1,2]. In particular, the tongue dorsum has long been considered a primary source of oral malodor [2-4], as it is reported that an effective means of alleviating oral malodor is through the active removal of bacteria from the tongue by using tongue scrapers of hard plastic or metal $[5,6]$. In fact, several investigators reported that oral malodor was associated with hydrogen sulfide-producing bacteria among tongue biofilm microflora [7-9], although VSC are produced from methionine and cysteine in mammalian tissue and by bacteria [10]. Detection of hydrogen sulfide is mainly done by four methods: colorimetric assays (mainly the methylene blue method), ion-selective electrode assays, high-performance liquid chromatography, and gas chromatography [10]. However, direct detection of hydrogen sulfide on the tongue dorsum remains difficult and the assessment of clinical oral malodour is usually done by measuring VSC levels in mouth breath using a gas chromatography-semiconductor gas sensor (Oral Chroma) and organoleptic scoring $[5,11]$. On the other hand, organoleptic assessment has been carried out using the spoon test by scraping the dorsal surface of the tongue, but without quantitative measurement $[3,4,12]$. Therefore, we tried to develop a method for visualization of the hydrogen sulfide on the tongue dorsum using methylene blue, which has been widely utilized for continuous, quantitative monitoring of hydrogen sulfide in biological samples 
[13-15]. However, the approach is problematic, making it less than ideal for measuring biological hydrogen sulfide. One issue is that the final product is blue and measured at $670 \mathrm{~nm}$, a wavelength at which significant interference by other colored substances has been demonstrated [13]. Consequently, complicated procedures have been necessary for methylene blue to be extracted from the reaction mixture by centrifugation and HPLC [15]. Thus, we attempted to develop a simple, inexpensive and novel colorimetric sensor system for direct detection of hydrogen sulfide on the tongue dorsum using a cotton swab and the methylene blue technique. We also investigated the relationship between the VSC levels in the breath monitored using the Oral Chroma and the content of hydrogen sulfide on the tongue dorsum.

\section{MATERIALS AND METHODS}

\subsection{Subjects}

Fourteen subjects (10 females and 4 males, mean age of 58.6 years; range, 24 - 81 years) were selected for this study. All subjects, who had no severe periodontal diseases and no bleeding (probing pocket depths of less than $3 \mathrm{~mm}$ ) and no systemic diseases, were patients who visited Hokkaido University Dental Hospital complaining of halitosis. On the first visit, an assessment of oral malodour and sampling of tongue biofilm were performed as described below. As reference subjects, 6 persons with no complaint of halitosis were selected from the staff (mean age of 40.3 years; range, 24 - 50 years) of the Department of Preventive Dentistry, Graduate School of Dental Medicine Hokkaido University, Sapporo, Japan. This project was approved by the Ethics Committee of the Hokkaido University Hospital.

\subsection{Materials}

$N, N^{\prime}$-dimethyl-p-phenylenediamine and zinc sulfate heptahydrate were purchased from Kanto Chemical Co., Japan, and Sigma-Aldrich Co., MO., USA, respectively. Ferric chloride, sodium sulfide and authentic standard hydrogen sulfide in toluene were purchased from Wako Pure Chemical Co., Japan. Plastic test tubes (15 ml) were obtained from TPP-US Corporation, MO., USA. All other chemicals were of the highest grade available commercially. Cotton swabs (length: $15.0 \pm 2.0 \mathrm{~mm}$; diameter: $4.7 \pm 0.6 \mathrm{~mm}$; capacity of volume retention: $120 \pm 10$ $\mu \mathrm{L}$ ) and cellulose chromatography paper (Advantec, No.526; thickness: $0.7 \mathrm{~mm}$; length: $25 \mathrm{~mm}$; diameter: 4.5 $\mathrm{mm}$; capacity of volume retention: $278 \pm 10 \mu \mathrm{L}$ ) were purchased from Sanritsu Co., Japan and Toyo Roshi Co., Japan, respectively.

\subsection{Analytical Methods for Measurement of Hydrogen Sulfide in the Gaseous Phase and Aqueous Solution}

Hydrogen sulfide was measured by three methods: gas chromatography-quadrupole mass spectrometry (GC-MS), with a gas chromatography-semiconductor gas sensor (Oral Chroma; Abimedical Co., Osaka, Japan), and the methylene blue colorimetric method. GC-MS was conducted using a QP5000 (Shimadzu, Tokyo, Japan), SPME fiber (Bellefonte, PA, USA), and Restek Stabilwax column (Restek, Bellefonte, PA, USA) as described previously [16]. The Oral Chroma, which is equipped with an indium oxide $\left(\operatorname{In}_{2} \mathrm{O}_{3}\right)$ semiconductor gas sensor, was used as described by Ansai et al. [17]. The methylene blue technique, which is based on Fischer's coupling reaction with $N, N$-dimethyl-p-phenylenediamine and ferric chloride [13], was used for measurement of hydrogen sulfide in the gaseous phase and aqueous solution.

\subsection{Preparation of Cotton Swab for Measurement of Hydrogen Sulfide}

A cotton swab was impregnated with $100 \mu \mathrm{L}$ of $20 \%$ zinc sulfate solution ( $\mathrm{w} / \mathrm{w}$ in distilled water) to preserve hydrogen sulfide, allowed to dry at room temperature for 1 hour and then lightly wrapped with aluminum foil and kept at room temperature. On the day of use, it was loaded with a cellulose paper column.

\subsection{Measurement of Hydrogen Sulfide in the Cotton Swab by the Methylene Blue Technique}

The hydrogen sulfide was first captured by zinc sulfate in the cotton swab. Next, the cotton swab was dipped into $30 \mu \mathrm{l}$ of $N, N^{\prime}$-dimethyl-p-phenylenediamine solution in a well of a disposable sample plate (No. 83-0357; Sansyo Co., Japan). After incubation for 30 seconds, it was further dipped into $15 \mu \mathrm{l}$ of $\mathrm{FeCl}_{3}$ solution and kept there for $5 \mathrm{~min}$. These procedures were carried out at room temperature. Subsequently methylene blue was produced by the reaction of $N, N^{\prime}$-dimethyl-p-phenylenediamine, ferric chloride and $\mathrm{HS}^{-}$at acidic $\mathrm{pH}$. The colored cotton swab was further dipped into $50 \mu \mathrm{l}$ of the stabilizing solution, which led to a distinct, stable color of methylene blue. After being photographed, the color density was analyzed using an optical device containing a light-emitting diode (LED) at a wavelength of $660 \mathrm{~nm}$ and a photodetector (Nippon Denshoku Co., Japan), as described previously [18]. The reaction solution for the methylene blue technique was prepared as follows: $\mathrm{FeCl}_{3}$ solution $(10 \mathrm{mg} / \mathrm{ml})$ was prepared in $1.2 \mathrm{M} \mathrm{HCl}$ solution and stored in a refrigerator. $N, N$ '-dimethyl-p-phenylenedia- 
mine solution $(100 \mathrm{mg} / \mathrm{ml})$ was prepared in ethyl alcohol containing $54 \mathrm{mg} / \mathrm{ml}$ thioglycerol, which stabilized the solution, and stored in a refrigerator. The solution was diluted with $0.1 \mathrm{M}$ Tris-HCl buffer ( $\mathrm{pH} 7.5$ ) to a $1 \mathrm{mg} / \mathrm{ml}$ concentration on the day of use. Sodium sulfide was used as the standard solution of hydrogen sulfide. Stock solution $(1.25 \mathrm{mM})$ was prepared by dissolving $0.748 \mathrm{~g}$ of $\mathrm{Na}_{2} \mathrm{~S} \cdot 9 \mathrm{H}_{2} \mathrm{O}$ in $100 \mathrm{ml}$ of distilled water and stored at $-20^{\circ} \mathrm{C}$. Working standards were prepared from the stock solution by appropriate dilution on the day of use. Stabilizing solution ( $\mathrm{pH} 4.5$ ) was prepared as follows: $10 \mathrm{ml}$ of $1 \mathrm{M} \mathrm{KH}_{2} \mathrm{PO}_{4}$ solution in distilled water, $1.0 \mathrm{ml}$ of $5 \%$ cetylpyrimidinium solution in distilled water, $3.3 \mathrm{ml}$ of $400 \mathrm{mM}$ ammonium chloride in $1 \mathrm{M} \mathrm{KH}_{2} \mathrm{PO}_{4}$, and $20 \mu \mathrm{l}$ of potassium permanganate solution $(20 \mathrm{mg} / \mathrm{ml})$ in $1 \mathrm{M}$ $\mathrm{KH}_{2} \mathrm{PO}_{4}$ were mixed and kept at room temperature.

\subsection{Assessment of Oral Malodor}

Oral malodor was measured by the principal investigator (TK) in two ways: detection of VSC in mouth air and organoleptic assessment. For whole-mouth malodor, the subjects were asked to breath out from the mouth at a distance of approximately $10 \mathrm{~cm}$ from the nose of the examiner [19]. Organoleptic assessment was estimated on a scale of 0 to 3 as follows: 0 , no odor; 1 , noticeable odor; 2, moderate odor; 3, strong odor. The VSC measurement in the mouth air was determined using an Oral Chroma, as described by Ansai et al. [17] and Miura et al. [20].

\subsection{Detection of Hydrogen Sulfide on the Tongue Dorsum}

Subjects refrained from oral intake and smoking for at least 2 hours before collection of tongue biofilm. After scraping the central region of the tongue dorsum ten times using a cotton swab impregnated with zinc sulfate, the hydrogen sulfide on the tongue dorsum was evaluated by the modified methylene blue technique as described above.

\subsection{Statistical Analysis}

Spearman's test was used for the statistical analysis. Data are expressed as mean \pm standard deviation. Regression analysis was used to determine the significance of the correlation between 2 changes.

\section{RESULTS}

\subsection{Identification of Hydrogen Sulfide Produced from Sodium Sulfide and Hydrogen Chloride}

To characterize the hydrogen sulfide produced from so- dium sulfide and hydrogen chloride in this experimental system, chemical analysis by GC-MS was conducted using a QP5000, SPME fiber, a Restek Stabilwax column, and authentic standard hydrogen sulfide. Twentyseven microliters of $0.2 \mathrm{mM}$ sodium sulfide was mixed with $27 \mu \mathrm{l}$ of $3.4 \mathrm{M} \mathrm{HCl}$ added using a microsyringe in a $15-\mathrm{ml}$ vial that was sealed with a screw cap at room temperature. After incubation for $5 \mathrm{~min}$ at room temperature, hydrogen sulfide in the gaseous phase was captured by the SPME fiber for $60 \mathrm{~min}$ at $50^{\circ} \mathrm{C}$. As shown in Figure 1, hydrogen sulfide produced in this experimental system was detected at 1.0 min of retention time in GC-MS analysis with helium gas, which was consistent with the authentic standard hydrogen sulfide. The GC-MS analysis also showed that the amount of hydrogen sulfide produced was $202.4 \pm 4.6 \mathrm{ng}(\mathrm{n}=3)$, which was almost same as the theoretical amount of the standard hydrogen sulfide (184 ng). Therefore, the results indicated that the system for production of hydrogen sulfide was reliable enough to use for the following experiments.

\subsection{Novel Assay System with Colorimetric Sensor for Direct Detection of Hydrogen Sulfide in Cotton Swab}

The hydrogen sulfide content in the cotton swab was evaluated by the modified methylene blue technique as shown in Figure 2. The methylene blue technique was

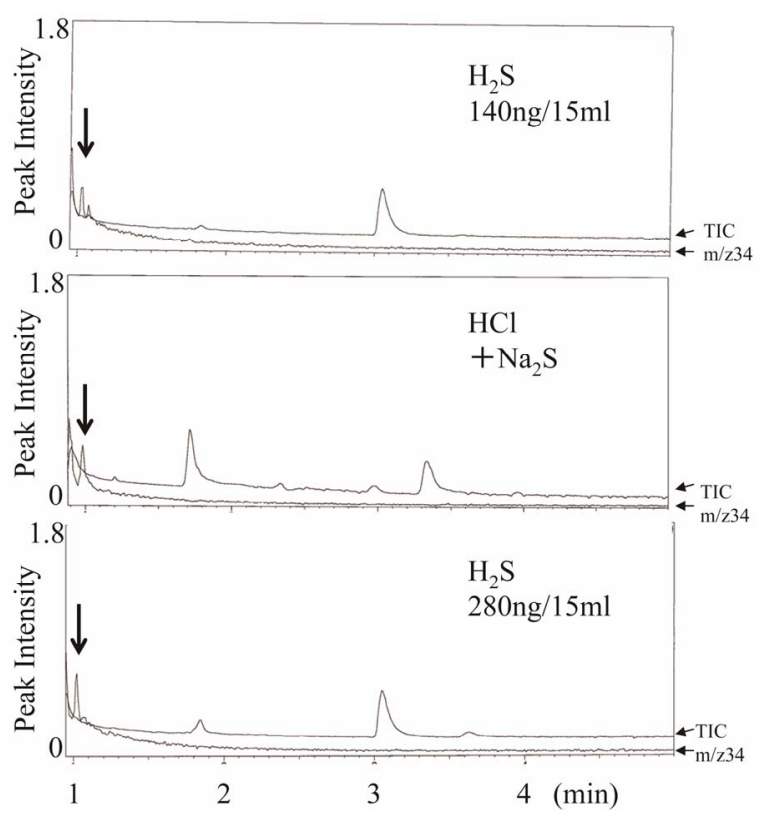

Figure 1. GC-MS chromatograms of standard hydrogen sulfide and synthesized hydrogen sulfide. Experimental conditions are described in the text. These ion chromatograms show total ion currents (TIC) and a mass chromatogram of the intact molecular ion of hydrogen sulfide $(\mathrm{m} / \mathrm{z}$ 34). Arrows indicate the peaks of hydrogen sulfide. 


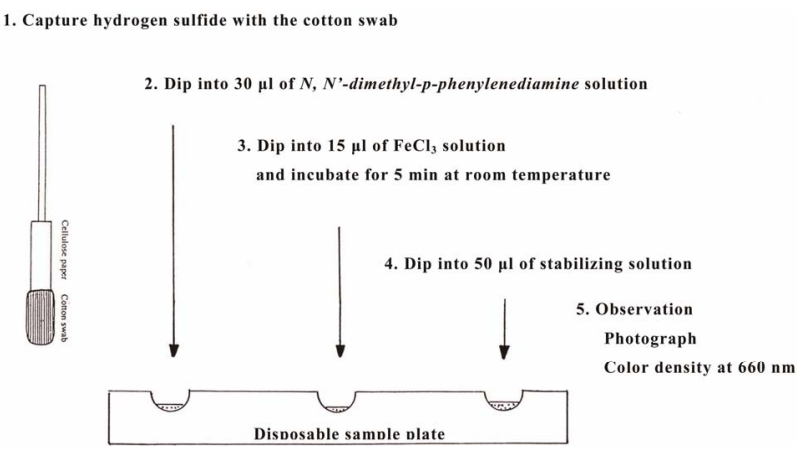

Figure 2. System for measurement of hydrogen sulfide in the cotton swab by the methylene blue technique. Experimental conditions are described in the text.

carried out in a cotton swab with $20 \mathrm{mg}$ of zinc sulfate using iron (III) and $N, N^{\prime}$-dimethyl-p-phenylenediamine at acidic $\mathrm{pH}$ in the optimal experimental conditions as described in the methods (data not shown). However, it produced many colored substances other than the methylene blue from hydrogen sulfide on it, as shown in Figure 3(a). Therefore, it was necessary to remove unreacted and produced substances other than the methylene blue from the cotton swab. We tried to separate the methylene blue from other reactants on the swab by trial and error, based on the characteristic of methylene blue technique. Attachment of a paper column led to removal of the brown color but did not show the distinct blue color (Figure 3(b)). On the other hand, with addition of $50 \mu$ of stabilizing solution there was a distinct blue color but brown was present as well (Figure 3(c)). Finally, with the attachment of the paper column and addition of stabilizing solution the distinct blue color was clear on the swab, whereas the chaotic brown color moved into the paper column (Figure 3(d)). These results confirmed the utility of the novel assay system with a colorimetric sensor for direct detection of hydrogen sulfide on the cotton swab.

\subsection{Relationship between Color Density of Methylene Blue and Hydrogen Sulfide Solution in Cotton Swab}

The potential of this method was assessed via the determination of the color density of methylene blue using a photodetector. As shown in Figure 4, a linear calibration graph was obtained between the concentrations of hydrogen sulfide and the absorbance at $660 \mathrm{~nm}$ for the methylene blue in the cotton swab. Using the methylene blue method it was confirmed that the detection limit of hydrogen sulfide was $5.3 \mathrm{ng}$ and the linearity was up to $85 \mathrm{ng}$. However, more than $85 \mathrm{ng}$ was outside of the linearity, showing that the absorbance was $0.188 \pm 0.037$ at the concentration of $85 \mathrm{ng}$ of hydrogen sulfide and 0.340 \pm 0.010 at $425 \mathrm{ng}$ (data not shown in Figure 4). Thus, the

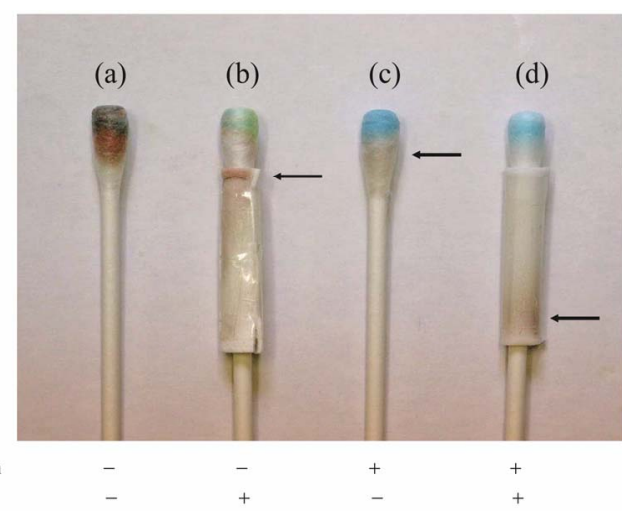

Paper column

Figure 3. Effects of the paper column and the stabilizing solution on the formation of methylene blue in the cotton swab. Experimental conditions are described in the text, except for the use of $42.5 \mathrm{ng}$ of hydrogen sulfide. Arrows indicate the chaotic color.

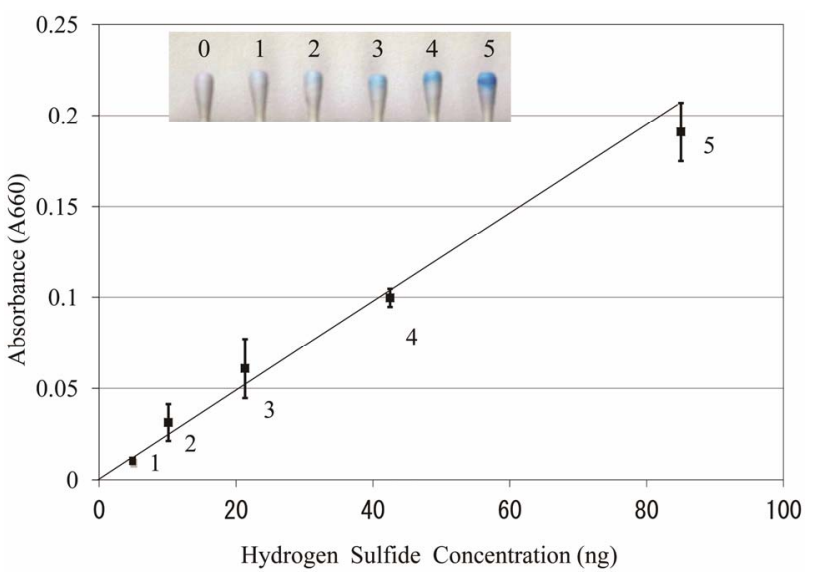

Figure 4. Relationship between color density of the methylene blue and hydrogen sulfide solution in the cotton swab. A solution of sodium sulfide was used as the standard for hydrogen sulfide. Other experimental conditions are described in the text. After taking the photograph (inset), the color density was analyzed at the wavelength of $660 \mathrm{~nm}$. The data represent the mean \pm SD from six experiments.

novel assay showed visually perceptible shades of color depending on the concentration of hydrogen sulfide.

\subsection{Time Course of Hydrogen Sulfide Captured by Cotton Swab in Gaseous Phase}

As described above, it was indicated that the novel assay system could be used as a visual warning sensor for assessment of oral malodor by using fluid samples. We next investigated the possibility of using it for the gaseous phase. Hydrogen sulfide in the gaseous phase was produced from sodium sulfide and hydrogen chloride in a $15 \mathrm{ml}$ vial as described above. The cotton swab was suspended from the cap to allow it to capture hydrogen sulfide at intervals of from $2 \mathrm{~min}$ to $180 \mathrm{~min}$ after mixture of two solvents, which were initially separately pre- 
sent in the bottom and on the side of the vial. The amount of hydrogen sulfide captured by the cotton swab increased depending on exposure time, recovering approximately $11 \%$ after $5 \mathrm{~min}$ and $90 \%$ after $180 \mathrm{~min}$ as compared to the total hydrogen sulfide. The relationship between the recovery of hydrogen sulfide and exposure time was almost the same at the concentrations of $230 \mathrm{ng}$ and $460 \mathrm{ng}$ (Figure 5). These results suggested that the assay system was not appropriate for assessment of hydrogen sulfide in the gaseous phase in a short period of time.

\subsection{Assessment of Hydrogen Sulfide on the Tongue Dorsum in Subjects with Complaints of Halitosis}

After scraping the central region of the tongue dorsum ten times using a cotton swab, hydrogen sulfide in the biofilm of the tongue dorsum was measured as described above. As shown in Figure 6, it showed different shades of methylene blue depending on the concentration of hydrogen sulfide in subjects with complaints of halitosis. Table 1 shows the relationship between hydrogen sulfide on the tongue dorsum and VSC levels in the breath determined using the Oral Chroma for the subjects with complaints of halitosis and reference subjects. The content of hydrogen sulfide on the tongue dorsum was significantly correlated with the hydrogen sulfide level monitored by the Oral Chroma $(\mathrm{r}=0.751, \mathrm{p}<0.01)$, but it was not detected in the subject showing the highest values of VSC $(20 \mathrm{ng} / 10 \mathrm{ml}$ hydrogen sulfide, $0.9 \mathrm{ng} / 10$ $\mathrm{ml}$ methylmercaptan, and $2 \mathrm{ng} / 10 \mathrm{ml}$ ). It also was positively associated with dimethyl sulfide $(\mathrm{r}=0.581, \mathrm{p}<$ $0.05)$ but not methyl mercaptan $(\mathrm{r}=0.167, \mathrm{p}=0.65)$. On the other hand, some reference subjects $(n=6)$ with no complaints of halitosis had little if any VSC in the breath

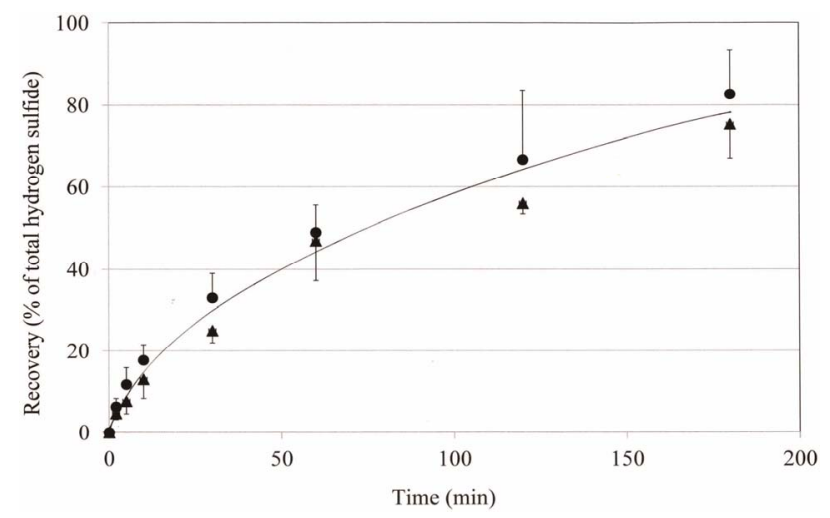

Figure 5. Time course of gaseous hydrogen sulfide capture by the cotton swab. Experimental conditions are described in the text. After exposure for various times, hydrogen sulfide captured by the cotton swab was measured as described in the text. The data represent $\%$ of recovery of the total hydrogen sulfide $(\mathrm{n}=3$, mean $\pm \mathrm{SD})$. Total amounts of hydrogen sulfide in 15 $\mathrm{ml}$ vials were $230 \mathrm{ng}(\boldsymbol{\Delta})$ and $460 \mathrm{ng}(\bullet)$, respectively.
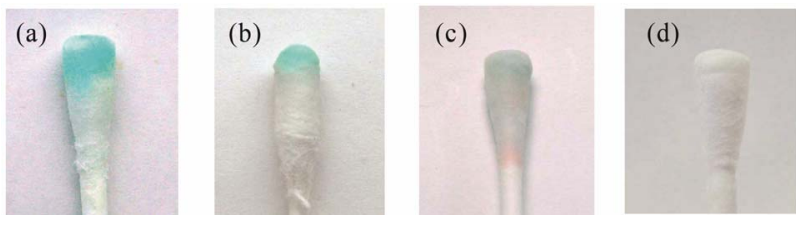

Figure 6. Typical presentations of hydrogen sulfide on the tongue dorsa from subjects with complaints of halitosis. Experimental conditions were described in Figure 4, except for using tongue dorsum scraping with the cotton swab. (a) $70 \mathrm{ng}$ of hydrogen sulfide on the tongue dorsum from a subject with a complaint of halitosis (VSC in the breath: $29.4 \mathrm{ng}$ of hydrogen sulfide, $7.8 \mathrm{ng}$ of methyl mercaptan, and $1.7 \mathrm{ng}$ of dimethyl sulfide); (b) $45 \mathrm{ng}$ of hydrogen sulfide on the tongue dorsum from a subject with a complaint of halitosis (VSC in the breath: 23.8, 5.3 and $6.8 \mathrm{ng}$, respectively); (c) $8 \mathrm{ng}$ of hydrogen sulfide on the tongue dorsum from a subject with a complaint of halitosis (VSC in the breath: 4.2, 2.3 and $1.3 \mathrm{ng}$, respectively); (d) $0 \mathrm{ng}$ of hydrogen sulfide on the tongue dorsum from a subject with a complaint of halitosis (VSC in the breath: 1.8, 0.4 and $1.3 \mathrm{ng}$, respectively).

or hydrogen sulfide on the tongue dorsum. These results indicated that hydrogen sulfide on the tongue dorsum reflected the generation of VSC hydrogen sulfide in the breath of the subjects with complaints of halitosis.

\section{DISCUSSION}

This is the first report on the visualization of the hydrogen sulfide on the tongue dorsum in subjects with complaints of halitosis. Numerous studies have previously found a relationship between the mechanical removal of the tongue coating and reduction of both the organoleptic score and VSC levels [5,11], as the coating (biofilm) on the tongue dorsum is recognized as a major source of VSC $[3,21]$. However, it has not been quantitatively demonstrated on the tongue dorsum, though it can be detected by smelling in the spoon test $[2,22]$ and by a tongue sulfide probe based on generation of electrochemical voltage [23]. Therefore, we attempted to develop a simple and novel colorimetric sensor for direct detection of hydrogen sulfide on the tongue dorsum, using the solid phase in a cotton swab and the methylene blue technique, although several investigators reported that methyl mercaptan and dimethyl sulfide are major causes of oral malodor compared to the hydrogen sulfide level $[24,25]$. Prior to measurement of hydrogen sulfide, we used $20 \mathrm{mg}$ of zinc sulfate per cotton swab to capture it, because the recommended dietary allowance of zinc is 40 - $45 \mathrm{mg} /$ day for adult men and 30 - $35 \mathrm{mg} /$ day for adult women in the Dietary Reference Intakes for Japanese [26]. Thus, there was no problem with safety for the healthy, even though scrapping might result in the transfer of zinc sulfate to the tongue. However, it remains to be determined whether zinc sulfate was transferred from the cotton swab to the tongue. 
Table 1. Comparison of hydrogen sulfide on the tongue dorsum and oral sulfur-containing gases in the breath of the subjects with complaints of halitosis and reference subjects.

\begin{tabular}{|c|c|c|c|c|c|}
\hline \multirow[t]{2}{*}{ Subject No. } & \multirow{2}{*}{$\begin{array}{l}\text { Hydrogen sulfide on the tongue } \\
\text { (ng/cotton swab) }\end{array}$} & \multicolumn{3}{|c|}{ Oral sulfur-containing gases (ng/10ml) } & \multirow[t]{2}{*}{ Organoleptic assessment } \\
\hline & & Hydrogen sulfide & Methyl mercaptan & Dimethyl sulfide & \\
\hline 1 & 32 & 29.4 & 15.4 & 7.8 & 3 \\
\hline 2 & 70 & 29.4 & 7.8 & 1.7 & 3 \\
\hline 3 & 45 & 23.8 & 5.3 & 6.8 & 3 \\
\hline 4 & 38 & 25.2 & 4.5 & 0.5 & 2 \\
\hline 5 & N.D. & 19.6 & 5.1 & 2.7 & 2 \\
\hline 6 & 19 & 13.2 & 0.7 & 6.4 & 2 \\
\hline 7 & 14 & 12.5 & 21.3 & 1.7 & 2 \\
\hline 8 & 22 & 10.1 & 7.5 & 3.8 & 2 \\
\hline 9 & 6 & 7.8 & 0.5 & 3.2 & 2 \\
\hline 10 & 11 & 6.5 & 1.3 & 8.9 & 1 \\
\hline 11 & 8 & 4.2 & 2.3 & 1.3 & 2 \\
\hline 12 & N.D. & 1.8 & 0.4 & 1.3 & 1 \\
\hline 13 & N.D. & 0.5 & 0.0 & 0.1 & 0 \\
\hline 14 & N.D. & 0.5 & 0.0 & 0.2 & 0 \\
\hline 1 & N.D. & 0.4 & 0.3 & 0.2 & 0 \\
\hline 2 & N.D. & 0.3 & 0.0 & 0.1 & 0 \\
\hline 3 & N.D. & 0.1 & 0.0 & 0.3 & 0 \\
\hline 4 & N.D. & 0.1 & 0.1 & 0.3 & 0 \\
\hline 5 & N.D. & 0.0 & 0.1 & 0.1 & 0 \\
\hline 6 & N.D. & 0.0 & 0.1 & 0.0 & 0 \\
\hline
\end{tabular}

Experimental conditions are described in the taet. N.D., not detected.

Regarding the methylene blue method, hydrogen sulfide captured by the cotton swab reacted with an equivalent of amount of $\mathrm{N}, \mathrm{N}$-dimethyl-p-phenylenediamine in the presence of ferric chloride at acidic $\mathrm{pH}$ and subsequently produced methylene blue. However, it was necessary to resolve two problems for measurement in the solid phase. First, the sensitivity had to be improved by a method optimized with respect to reagent strength and $\mathrm{pH}$ because the appearance of methylene blue in the final product is affected by these two factors, detergent, and oxidants $[15,27,28]$. Second, the reaction also produces other colored substances that interfere with the measurement of methylene blue as pointed out by Siegel [13]. To overcome these problems, we found the optimal experimental conditions for the methylene blue technique with respect to reagent strength, and $\mathrm{pH}$ by trial and error (data not shown). Addition of thioglycerol, an antioxidant, to the $N, N$-dimethyl-p-phenylenediamine solution improved the recovery of hydrogen sulfide in the assay system by $144 \%$ (data not shown). Use of the stabilizing solution, which was prepared based on the chemical characteristics of methylene blue [27,29], led to distinct methylene blue color in the cotton swab. Attachment of the paper column resulted in removal of the chaotic color from the cotton swab via the theory of paper chromatog- raphy. Thus, the paper column and a stabilizing solution improved the stability and the sensitivity of the methylene blue formed in the cotton swab, so that it was adsorbed into the cotton swab but other reactants were moved out it. However, it is not clear by which mechanism the stabilizing solution improved the stability and the sensitivity of the methylene blue in the cotton swab. Thus, this point should be further investigated.

The assay system demonstrated linearity up to $85 \mathrm{ng}$ for direct detection of hydrogen sulfide in the cotton swab, with a detection limit of $5.3 \mathrm{ng}$. Unexpectedly, the assay system for the gaseous phase of hydrogen sulfide was less sensitive than that of solution in the solid phase because the cotton swab required a long period of time to capture gaseous hydrogen sulfide. The recovery of hydrogen sulfide was only $11 \%$ of total hydrogen sulfide in $5 \mathrm{~min}$, whereas the gas chromatography-semiconductor gas sensor (Oral Chroma) could measure gaseous hydrogen sulfide immediately $[17,20]$. These results indicated that the assay system was not appropriate for assessment of gaseous hydrogen sulfide in the whole mouth.

Next, we tried to evaluate visualization of the hydrogen sulfide by scraping the central region of the tongue dorsum because the sulfide level and biofilm matrix were mostly located in it $[23,30]$. Scraping was carried out in 
this region of the tongue dorsum as it was most readily accessed and was not affected by residual saliva. In a preliminary experiment using the control subjects the content of water trapped by the cotton swab was constant, $0.036 \pm 0.014 \mathrm{~g}(\mathrm{n}=8)$, although it is well known that the coating on the dorsum of the tongue is comprised of two fluidic compartments, salivary film and biofilm matrix fluid. Thus it was confirmed that scraping did not affect the flow rate of fresh saliva. As expected, the assay system showed various shades of methylene blue depending on the concentration of hydrogen sulfide on the tongue dorsum in subjects with complaints of halitosis. Interestingly, it was previously reported that the tongue dorsum was a major source of hydrogen sulfide and methyl mercaptan production $[8,31]$. Therefore, the color density of methylene blue seems to reflect the concentration of hydrogen sulfide on the tongue dorsum. However, the present method cannot differentiate free hydrogen sulfide causing halitosis from bound hydrogen sulfide (acid-labile sulfide) such as iron-sulfur clusters of non-heme iron-sulfur proteins, which release free hydrogen sulfide under acidic conditions [10,32], because the biofilm of the tongue dorsum is comprised of desquamated epithelial cells, blood cells, metabolites, nutrients and bacteria [24].

Finally, we examined the relationship between the VSC level obtained with the Oral Chroma and the content of hydrogen sulfide on the tongue dorsum in order to develop a visual warning sensor for halitosis. Regarding the clinical reliability and precision of the Oral Chroma, several reports suggest that it has sensitivity and specificity very similar to those of the Halimeter [33-35]. The content of hydrogen sulfide on the tongue dorsum was positively associated with the levels of hydrogen sulfide and methyl mercaptan in the breath monitored by the Oral Chroma but not with dimethyl sulfide. Regarding the comment that dimethyl sulfide cannot be detected by the Oral Chroma, Ansai et al. and Tsai et al. previously reported that it was detected in mouth air from patients by the Oral Chroma [17,36]. However, hydrogen sulfide on the tongue dorsum was not observed in one of the subjects with higher VSC hydrogen sulfide. This discrepancy may have occurred because the methylene blue technique is very tricky and is subject to interference by several substances [28]. Recently, Shen et al. also pointed out that the protein concentration in the measured biological sample affected the value of hydrogen sulfide [15]. These problems should be dealt with to obtain higher detection sensitivity in the future. However, the assay system was specific, reflecting the content of hydrogen sulfide and can be used as a visual warning sensor for oral malodor by measuring hydrogen sulfide on the tongue dorsum, even though the hydrogen sulfide could not quantitatively evaluated.

\section{REFERENCES}

[1] Tonzetich, J. (1977) Production and origin of oral malodor: A review of mechanisms and methods of analysis. Journal of Periodontology, 48, 13-20. doi:10.1902/jop.1977.48.1.13

[2] Rosenberg, M. (1996) Clinical assessment of bad breath: Current concepts. Journal of American Dental Association, 127, 475-482.

[3] De Boever, E.H. and Loesche, W.J. (1995) Assessing the contribution of anaerobic microflora of the tongue to oral malodor. Journal of American Dental Association, 126, 1384-1392.

[4] Bosy, A., Kulkarmi, G.V., Rosenberg, M., et al. (1994) Relationship of oral malodor to periodontotitis: Evidence of independence in discrete subpopulations. Journal of Periodontology, 65, 37-46. doi:10.1902/jop.1994.65.1.37

[5] Cicek,Y., Orbak, R., Tezel, A., et al. (2003) Effect of tongue brushing on oral malodor in adolescents. Pediatrics International, 45, 719-723. doi:10.1111/j.1442-200X.2003.01804.X

[6] Christensen, G.J. (1998) Why clean your tongue? Journal of American Dental Association, 129, 1605-1607.

[7] Haraszthy, V.I., Zambon, J.J., Sreeivasan, P.K., et al. (2007) Identification of oral bacterial species associated with halitosis. Journal of American Dental Association, 139, 1113-1120.

[8] Washio, J., Sato, T., Koseki, T., et al. (2005) Hydrogen sulfide-producing bacteria in tongue biofilm and their relationship with oral malodour. Journal of Medical Microbiology, 54, 889-895. doi:10.1099/jmm.0.46118-0

[9] Kazor, C.E., Mitchell, P.M., Lee, A.M., et al. (2003) Diversity of bacterial populations on the tongue dorsa of patients with halitosis and healthy patients. Journal of Clinical Microbiology, 41, 558-563. doi:10.1128/JCM.41.2.558-563.2003

[10] Tangerman, A. (2009) Measurement and biological significance of the volatile sulfur compounds hydrogen sulfide, methanethiol and dimethyl sulfide in various biological matrices. Journal of Chromatography, B877, 33663377. doi:10.1016/j.jchromb.2009.05.026

[11] Tsai, C.C., Chou, H.H., Wu, T.L., et al. (2008) The levels of volatile sulfur compounds in mouth air from patients with chronic periodontitis. Journal of Periodontal Research, 43, 186-193. doi:10.1111/j.1600-0765.2007.01011.x

[12] Greenstein, R.B., Goldberg, S., Marku-Cohen, S., et al. (1997) Reduction of oral malodour by oxidizing lozenges. Jounal of Periodontology, 68, 1176-1181. doi:10.1902/jop.1997.68.12.1176

[13] Siegel, L.M. (1965) A direct microdetermination for sulfide. Analytical Biochemistry, 11, 126-132. doi:10.1016/0003-2697(65)90051-5

[14] Zhong, G., Yang, X., Jia, L., et al. (2009) Inhibition of the cystathione- $\gamma$-lyase/hydrogen sulfide pathway in rat vascular smooth muscle cells by cobalt- 60 gamma radiation. Chinese Medical Journal, 122, 326-330.

[15] Shen, X., Pattillo, C.B., Pardue, S., et al. (2011) Meas- 
urement of plasma hydrogen sulfide in vivo and in vitro. Free Radical Biology and Medicine, 50, 1021-1031. doi:10.1016/j.freeradbiomed.2011.01.025

[16] Osada, K., Curran, M., Kurachi, M., et al. (2008) Effect of an orally ingested mugwort and mushroom extract mixture on urine odor from aged mice. Bioscience, Biotechnology, and Biochemistry, 72, 1249-1256. doi:10.1271/bbb.70718

[17] Ansai, T., Awano, S., Soh, I., et al. (2009) Associations among hair loss, oral sulfur-containing gases, and gastrointestinal and metabolic linked diseases in Japanese elderly men: Pilot study. BMC Public Health, 9, 82. doi:10.1186/1471-2458-9-82

[18] Sakamoto, W., Fujii, Y., Kanehira, T., et al. (2008) A novel assay system for myeloperoxidase activity in whole saliva. Clinical Biochemistry, 41, 584-590. doi:10.1016/j.clinbiochem.2007.12.025

[19] Rosenberg, M., Kozlovsky, A., Gelernter, I., et al. (1995) Self-estimation of oral malodor. Journal of Dental Research, 74, 1577-1582. doi:10.1177/00220345950740091201

[20] Miura, T., Rahardjo, A., Fujiwara, Y., et al. (2006) Development of a compact and simple gas chromatography for oral malodor measurement. Journal of Periodontology, 77, 1142-1147. doi:10.1902/jop.2006.050388

[21] Yaegaki, K. and Sanada, K. (1992) Volatile sulfur compounds in mouth air from clinically healthy subjects and patients with periodontal disease. Journal of Periodontal Research, 27, 233-238. doi:10.1111/j.1600-0765.1992.tb01673.x

[22] Greenstein, R.B., Goldberg, S., Marku-Cohen, S., et al. (1997) Reduction of oral malodour by oxidizing lozenges. Journal of Periodontology, 68, 1176-1181. doi:10.1902/jop.1997.68.12.1176

[23] Morita, M., Musinski, D.L. and Wang, H.L. (2001) Assessment of newly developed tongue sulfide probe for detecting oral malodor. Journal of Clinical Periodontology, 28, 494-496. doi:10.1034/j.1600-051x.2001.028005494.x

[24] Tangerman, A. and Winkel, E.G. (2007) Intra- and extra-oral halitosis: Finding of a new form of extra-oral blood-borne halitosis caused by dimethyl sulphide. Journal of Clinical Periodontology, 34, 748-755. doi:10.1111/j.1600-051X.2007.01116.x

[25] Awano, S., Koshimura, S., Kurihara, E., et al. (2004) The assessment of methyl mercaptan, an important clinical marker for the diagnosis of oral malodor. Journal of Den- tistry, 32, 555-559. doi:10.1016/j.jdent.2004.06.001

[26] The National Nutrition Survey in Japan (2010) Ministry of Health, Labour and Welfare, Tokyo, Japan.

[27] Pandurangappa, M. and Samrat, D. (2010) Micellarmediated extractive spectrophotometric determination of hydrogen sulfide/sulfide through Prussian blue reaction: Application to environmental samples. Analytical Science, 26, 83-87. doi:10.2116/analsci.26.83

[28] APHA (American Public Health Association) (1998) Standard Methods for the Examination of Water and Wastewater, 20th ed.; APHA, American Water Works Association, and Water Pollution Control Federation, Washington DC, USA.

[29] Janos, P. (2003) Sorption of basic dyes onto iron humate. Environmental Science and Technology, 37, 5792-5798. doi:10.1021/es020142o

[30] Roldan, S., Herrera, D. and Sanz, M. (2003) Biofilms and the tongue: Therapeutical approaches for the control of halitosis. Clinical Oral Investigations, 7, 189-197. doi:10.1007/s00784-003-0214-7

[31] Krespi, Y.P., Schrime, M.G., and Kacker, A. (2006) The relationship between oral malodor and volatile sulfur compound-producing bacteria. Otolaryngology-Head and Neck Surgery, 135, 671-676. doi:10.1016/j.otohns.2005.09.036

[32] Ubuka, T. (2002) Assay methods and biological roles of labile sulfur in animal tissues. Journal of Chromatography, B781, 227-249. doi:10.1016/S1570-0232(02)00623-2

[33] Vandekerckhove, B., Van den Velde, S., De Smith, M., et al. (2009) Clinical reliability of non-organoleptic oral malodour measurements. Journal of Clinical Periodontology, 36, 964-969. doi:10.1111/j.1600-051X.2009.01473.X

[34] Salako, N.O. and Philip, L. (2011) Comparison of the use of the Halimeter and the Oral Chroma ${ }^{\mathrm{TM}}$ in the assessment of the ability of common cultivable oral anaerobic bacteria to produce malodorous volatile sulfur compounds from cysteine and methionine. Medical Principles and Practical, 20, 75-79. doi:10.1159/000319760

[35] Pham, T.A. (2012) Comparison between self-estimated and clinical oral malodor. Acta Odontologia Scandinavia, March 29.

[36] Tsai, C.C., Chou, H.H., Wu, T.L., et al. (2008) The levels of the volatile sulfur compounds in mouth air from patients with chronic periodontitis. Journal of Periodontal Research, 43, 186-193. doi:10.1111/j.1600-0765.2007.01011.x 